\title{
Joint Thrombus and Vessel Segmentation Using Dynamic Texture Likelihoods and Shape Prior
}

\author{
Nicolas Brieu ${ }^{1}$, Martin Groher ${ }^{1}$, Jovana Serbanovic-Canic ${ }^{2,3}$, \\ Ana Cvejic ${ }^{2,3}$, Willem Ouwehand ${ }^{2,3,4}$, and Nassir Navab ${ }^{1}$ \\ 1 Computer Aided Medical Procedures, Technische Universität München, Germany \\ brieu@in.tum.de \\ 2 The Wellcome Trust Sanger Institute, Hinxton, UK \\ 3 Department of Hematology, University of Cambridge, UK \\ 4 NHS Blood and Transplant, Cambridge, UK
}

\begin{abstract}
The segmentation of thrombus and vessel in microscopic image sequences is of high interest for identifying genes linked to cardiovascular diseases. This task is however challenging because of the low contrast and the highly dynamic conditions observed in time-lapse DIC in-vivo microscopic scenes. In this work, we introduce a probabilistic framework for the joint segmentation of thrombus and vessel regions. Modeling the scene with dynamic textures, we derive two likelihood functions to account for both spatial and temporal discrepancies of the motion patterns. A tubular shape prior is moreover introduced to constrain the aortic region. Extensive experiments on microscopic sequences quantitatively show the good performance of our approach.
\end{abstract}

\section{Introduction}

Thrombosis refers to the aggregation of blood cells occurring after a blood vessel injury. The analysis of thrombus formation in mutant zebrafish larvae is becoming crucial to identify genes related to cardiovascular diseases [11. The following experiment is conducted: the specimen is put under a microscope, the wall of its caudal aorta is artificially injured to initiate thrombosis, and images are recorded using a microscope/camera system. Measures such as time to attachment of the first blood cell, thrombus surface area, and time to occlusion of the aorta, are manually computed to characterize the thrombus formation. Since such experiments are repeated many times to conduct statistical studies, the characterization needs to be automated. This can be achieved by developing algorithms for the segmentation of the thrombus and of the aortic regions.

Segmenting in vivo microscopic images is challenging. As illustrated by previous works on microscopic image segmentation 6913, common intensity-based or gradient-based methods can hardly be applied because of the low image quality and the cluttered environment of in vivo scenes. This is particularly true in our application (cf. Fig. 1a). The low contrast between the thrombus and the aorta challenges the computation of intensity-related features. The number of blood cell or streamline-related clutters in the aorta hampers the derivation of

G. Fichtinger, A. Martel, and T. Peters (Eds.): MICCAI 2011, Part III, LNCS 6893, pp. 579-586 2011.

(C) Springer-Verlag Berlin Heidelberg 2011 

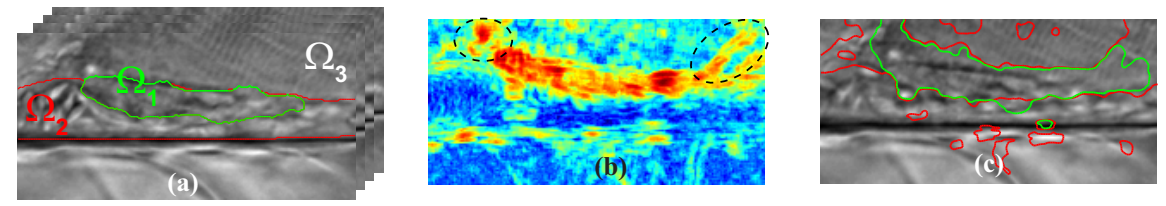

Fig. 1. (a) Microscopic image sequence and Ground-truth segmentation; (b) Residual energy from DT modeling [4]; (c) Intensity-based segmentation of (b)

gradient-related cues. As such, motion seems to be a better discriminative cue. To account for the non-homogeneity of biological objects which hampers the use of first-order intensity-related features, a motion-related flux tensor derived from optical-flow computation has been introduced in [12. In our case, however, the chaotic movement of the blood cells in the uncoagulated aorta contradicts the brightness constancy assumption made for optical-flow derivation. As such, and similarly to [3], we favor the Dynamic Texture (DT) approach of Doretto et al. 7] that provides reliable motion-related cues in similarly complex scenarios [4] (Fig. 11p-c).

Our previous work [3] has two main limitations. First, it is restricted to the segmentation of the thrombus region. Second, it is disrupted by the slow circulation of blood cells in the collateral vessels and by the local non-rigid movements in the background. In this paper, we propose an approach which overcomes these limitations. We derive likelihood and prior energies from the following observations. (1) The thrombus, the uncoagulated aorta, and the background are characterized by distinct motion patterns. We model the spatial homogeneity of the motion patterns in each region with a motion-segmentation likelihood energy. (2) The thrombus is the only region which does not exist at the initial state of the sequence, i.e. before the attachment of the first blood cell. We introduce a second likelihood energy, the event-detection energy, which characterizes the variations of the motion patterns between the initial and the current states. (3) The spatial extents of the regions are constrained: the aorta resembles a tubular shape and the thrombus is included in the aorta. By modeling these constraints as a shape prior energy, we counter-balance the collateral vessels and further increase the accuracy of the segmentations.

The key contributions of this paper are the introduction of the event-detection likelihood energy, the definition of the shape prior energy, and the quantitative validation of our method on in vivo microscopic sequences against manually delineated ground truth segmentations. Experiments show the improvements in accuracy induced by the two newly developed energies.

\section{Method}

Let us consider a video sequence $\mathcal{S}=\left\{\mathcal{I}^{p}, 0 \leq p<T\right\}$. For each time $p$, our objective is to partition the image domain $\Omega$ into the thrombus $\left(\Omega_{1}\right)$, the uncoagulated aorta $\left(\Omega_{2}\right)$, and the background region $\left(\Omega_{3}\right)$ (Fig $[1$ a). The reunion 
of the thrombus and of the uncoagulated aortic regions forms the aortic region, $\Omega_{12}=\Omega_{1} \cup \Omega_{2}$. To assess the temporal information necessary to define the two likelihood energies, we consider the sub-sequence of $\tau$ frames centered at time $p, \mathcal{S}^{p}=\left\{\mathcal{I}^{t}, p-\tau / 2 \leq t \leq p+\tau / 2\right\}$, and also the initial sub-sequence $S^{0}$. Finding the optimal partition $\omega^{*}$ of the image domain at time $p$ given $\mathcal{S}^{p}$ and $\mathcal{S}^{0}$ is formulated as an energy minimization problem

$$
\omega^{*}=\arg \min _{\omega}\left[E\left(\mathcal{S}^{p}, \mathcal{S}^{0} \mid \omega\right)+E(\omega)\right] .
$$

The likelihood energy $E\left(\mathcal{S}^{p}, \mathcal{S}^{0} \mid \omega\right)$ regroups both the motion-segmentation and the event-detection energies. The prior energy $E(\omega)$ constrains the partition.

\subsection{Dynamic Texture Likelihoods}

The definition of motion-based segmentation likelihoods can be decomposed in a series of successive steps. We have to select a motion model, to identify for each pixel the model parameters corresponding to the observed motion pattern, and to define a metric on the model parameter subspace. This permits the relative comparison of the motion patterns observed in the scene and the introduction of likelihood functions accounting for their homogeneity in space and in time.

Dynamic Texture Model. Let us consider the sub-sequence $\mathcal{S}^{p}$ and the square spatial patch of $m$ pixels centered at the pixel $\mathbf{x}$. The intensities $\mathbf{y}(t) \in \mathbb{R}^{m}$ observed in the patch at a time $t$ are related to a set of hidden variables $\mathbf{z}(t) \in \mathbb{R}^{n}$ by the linear dynamic system $\mathbf{y}(t)=\mathbf{C z}(t)+\mathbf{w}(t)$ and $\mathbf{z}(t+1)=\mathbf{A} \mathbf{z}(t)+\mathbf{v}(t)$ [7]. The first equation describes the simplification of the intensities in a set of $n<<m$ spatial features. The second equation accounts for the evolution of these features over time. $\mathrm{A} \in \mathbb{R}^{n \times n}$ and $\mathrm{C} \in \mathbb{R}^{m \times n}$ are the state-transition and the observation matrices. The two normal random processes $\mathbf{v}(t) \propto \mathcal{N}(0, \mathbf{Q}), \mathbf{Q} \in \mathbb{R}^{n \times n}$, and $\mathbf{w}(t) \propto \mathcal{N}(0, R), \mathrm{R} \in \mathbb{R}^{m \times m}$, account for noise in the hidden and in the observed states respectively. Stacking the system for the $\tau$ temporal states $(t=1 \ldots \tau)$ of $\mathcal{S}^{p}$ yields the matrix equations $\mathrm{Y}_{1}^{\tau}=\mathrm{CZ}_{1}^{\tau}+\mathrm{W}$ and $\mathrm{Z}_{2}^{\tau}=\mathrm{AZ}_{1}^{\tau-1}+\mathrm{V}$ where $\mathrm{Z}_{p}^{q}=[\mathbf{z}(p), \ldots, \mathbf{z}(q)]$ and $\mathrm{Y}_{p}^{q}=[\mathbf{y}(p), \ldots, \mathbf{y}(q)]$. Neglecting the noise processes, an approximate set of parameters $\mathcal{D}_{\mathbf{x}}=\{\mathrm{A}, \mathrm{C}\}$ is found by constraining $\mathrm{C}$ to be orthogonal [7]. Given the singular value decomposition $\mathrm{Y}_{1}^{\tau}=\mathrm{U \Sigma V}^{T}$, the observation matrix, the hidden variables, and the state-transition matrix read as $\hat{\mathrm{C}}=\mathrm{U}$, $\hat{\mathrm{Z}_{1}^{\tau}}=\Sigma \mathrm{V}^{T}$, and $\hat{\mathrm{A}}=\hat{\mathrm{Z}}_{2}^{\tau}\left(\hat{\mathrm{Z}}_{1}^{\tau-1}\right)^{\dagger}$ respectively. $(.)^{\dagger}$ denotes the pseudoinverse.

Distance. We calculate at each pixel $\mathbf{x} \in \Omega$ the DT models $\mathcal{D}_{\mathbf{x}}^{0}$ and $\mathcal{D}_{\mathbf{x}}^{p}$ for the initial sub-sequence $\mathcal{S}^{0}$ and for the current sub-sequence $\mathcal{S}^{p}$. In [3], each DT model is represented by the ratio between the norm of the prediction matrix $\left\|A Z_{1}^{\tau-1}\right\|_{2}$ and the norm of the residual matrix $\left\|\mathrm{Z}_{2}^{\tau}-\mathrm{AZ}_{1}^{\tau-1}\right\|_{2}$. In this case, the distance between the DT models is defined as the Euclidean distance between the ratios. However, and as illustrated in Fig 1 b, this simplified representation hampers the distinction between the thrombus and collateral vessels, and between the aorta and the nearly static background. We instead maintain the 
(a)

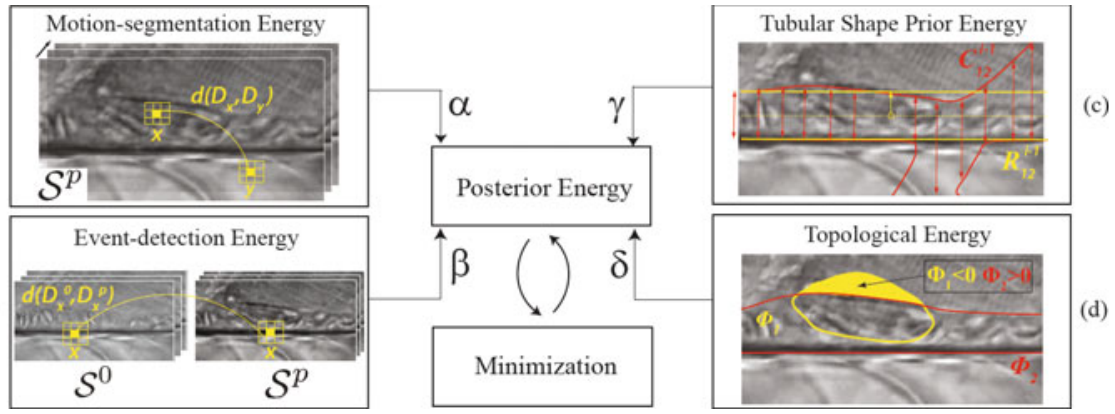

Fig. 2. Proposed framework for joint thrombus-vessel segmentation

complete parametrization of the DT models with their respective matrices A and $\mathrm{C}$. The orthogonality constraint on the matrix $\mathrm{C}$ yields the non-linearity of the DT model manifold. A non-linear similarity measure on this manifold thus becomes necessary. Similarly to [8], we choose to employ the Martin distance [10] because of its good precision and of its simple computation.

Motion Segmentation Energy. Analyzing the spatial variations of the DT models at the sub-sequence $\mathcal{S}^{p}$ is justified by the discrepancy of the motion patterns between the different regions: the thrombus is slowly moving synchronously with the heart beat, the background is nearly static, and the uncoagulated aorta is characterized by the fast and chaotic circulation of blood cells.

Let us assume that the DT models $\left\{\mathcal{D}_{\mathbf{x}}, \mathbf{x} \in \Omega_{k}\right\}$ in each region $\Omega_{k}$ follow a normal distribution $\mathcal{N}\left(\mu_{\Omega_{k}}, \sigma_{\Omega_{k}}\right)$ with respect to the Martin distance $d$. Extending the probabilistic approach of intensity-based segmentation [5] to dynamic texture segmentation, the motion segmentation likelihood energy is defined by

$$
E_{m s}\left(\mathcal{S}^{p}, \mathcal{S}^{0} \mid \omega\right)=\sum_{k=1}^{3} \sum_{\mathbf{x} \in \Omega_{k}} \frac{d^{2}\left(\mathcal{D}_{\mathbf{x}}, \mu_{\Omega_{k}}\right)}{2 \sigma_{\Omega_{k}}^{2}} .
$$

Because of the non-Euclidean structure of the DT model space, the mean model $\mu_{\Omega_{k}}$ and the standard deviation $\sigma_{\Omega_{k}}$ cannot be easily computed. The squared Martin distance $d^{2}\left(\mathcal{D}_{\mathbf{x}}, \mu_{\Omega_{k}}\right)$ is thus approximated by its expected value. Given a set of DT model samples $\left\{\mathcal{D}_{\mathbf{y}}, \mathbf{y} \in \Omega_{k}^{s}\right\}$ computed from a set of randomly selected pixel samples $\Omega_{k}^{s}$, the expected value and the standard deviation read as

$$
\hat{d}^{2}\left(\mathcal{D}_{\mathbf{x}}, \mu_{\Omega_{k}}\right)=\frac{1}{\left|\Omega_{k}^{s}\right|} \sum_{\mathbf{y} \in \Omega_{k}^{s}} w_{k, \mathbf{y}} d^{2}\left(\mathcal{D}_{\mathbf{x}}, \mathcal{D}_{\mathbf{y}}\right), \hat{\sigma}_{\Omega_{k}}^{2}=\frac{1}{\left|\Omega_{k}\right|} \sum_{\mathbf{x} \in \Omega_{k}} \hat{d}^{2}\left(\mathcal{D}_{\mathbf{x}}, \mu_{\Omega_{k}}\right) .
$$

Estimating the standard deviation of the DT model distribution allows us to normalize the likelihood energy. The tractability of Eq. (3) is ensured by choosing $\left|\Omega_{k}^{s}\right|<<\left|\Omega_{k}\right|$. To prevent abrupt change of the likelihood energy if a peripheral sample exits the region, the samples in the center of the region are favored by setting $w_{k, \mathbf{y}}$ equal to the Euclidean distance of $\mathbf{y}$ to the boundaries of $\Omega_{k}$. 
Event Detection Energy. In addition to its distinct motion pattern, the thrombus is characterized by its growth in a previously uncoagulated aorta. We model this observation by analyzing the variations of the local motion patterns between the initial sub-sequence $\mathcal{S}^{0}$ and the current sub-sequence $\mathcal{S}^{p}$.

Let us first consider a pixel $\mathbf{x}$ belonging to the thrombus region in $\mathcal{S}^{p}$. This pixel belongs to the uncoagulated aortic region in $\mathcal{S}^{0}$. Its motion pattern is modified. The distance between the DT model $\mathcal{D}_{\mathbf{x}}^{0}$ observed in $\mathcal{S}^{0}$ and the DT model $\mathcal{D}_{\mathbf{x}}^{p}$ observed in $\mathcal{S}^{p}$ is thus significant. Please note that the recording is started before the laser injury to ensure that the thrombus has not formed in the initial subsequence $\mathcal{S}_{0}$. Let us then consider a pixel $\mathbf{x}$ belonging to the uncoagulated aorta or to the background region in $\mathcal{S}^{p}$. Following the observation that the aorta boundaries remain nearly constant, the pixel $\mathbf{x}$ belongs to the same region in $\mathcal{S}^{0}$. Its motion pattern is unchanged and the distance between $\mathcal{D}_{\mathbf{x}}^{0}$ and $\mathcal{D}_{\mathbf{x}}^{p}$ tends to zero. We define the event-detection map $\Delta_{\mathbf{x}}^{0, p}$ in each pixel $\mathbf{x}$ as the Martin distance between the DT models $\mathcal{D}_{\mathbf{x}}^{0}$ and $\mathcal{D}_{\mathbf{x}}^{p}$. This map is characterized by high values for the thrombus region and by low values for the uncoagulated aorta and the background region.

Assuming a normal distribution $\mathcal{N}\left(\Delta_{\Omega_{k}}^{0, p}, \sigma_{\Omega_{k}}^{0, p}\right)$ of the $\Delta_{\mathbf{x}}^{0, p}$ in the $\mathrm{k}^{\text {th }}$ region, we encourage the homogeneity of the partition with respect to the temporal changes of the DT models by introducing the event-detection energy

$$
E_{e d}\left(\mathcal{S}^{p}, \mathcal{S}^{0} \mid \omega\right)=\sum_{k=1}^{3} \sum_{\mathbf{x} \in \Omega_{k}} \frac{\left(\Delta_{\mathrm{x}}^{0, p}-\Delta_{\Omega_{k}}^{0, p}\right)^{2}}{2\left(\sigma_{\Omega_{k}}^{0, p}\right)^{2}} \text {, with } \Delta_{\mathrm{x}}^{0, p}=d\left(\mathcal{D}_{\mathrm{x}}^{0}, \mathcal{D}_{\mathrm{x}}^{p}\right) \text {. }
$$

The likelihood energies defined by Eq.(2) and Eq.(4) characterize the spatial and temporal changes of the DT models. However, and as shown in Fig 3 c, these energies and the subsequent segmentation results are disrupted by the slow circulation of blood cells in the collateral vessels and by the local non-rigid movements of the background regions. A prior energy thus becomes necessary.

\subsection{Tubular Shape Prior Energy}

The collateral vessels and the non-rigid movements in the background deform the aorta segmentation towards a non-tubular structure (Fig 3 r). We constrain the shape of the aortic region $\Omega_{12}$ by introducing a tubular shape prior energy $E_{s p}(\omega)$ into our segmentation framework (Fig 2r). At each iteration $i$ of the energy minimization scheme, we fit a rectangle $\mathcal{R}_{12}^{i-1}$ to the contour $\left(\mathcal{C}_{12}^{i-1}\right)$ of the aortic region, taken at the previous iteration. We then define

$$
E_{s p}(\omega)=\sum_{k=1}^{2} \sum_{x \in \Omega_{k}}\left[1-\exp \left(-\frac{d_{R_{12}^{i-1}}^{2}(\mathbf{x})}{2 \sigma^{2}}\right)\right] .
$$

$d_{R_{12}^{i-1}}($.$) denotes the distance transform of the binary image derived from R_{12}^{i-1}$. $\sigma \in \mathbb{R}$ sets the range of feasible deformations. The centerline of $R_{12}^{i-1}$ is approximated by the principal axis of $\mathcal{C}_{12}^{i-1}$, and its radius by the median distance 

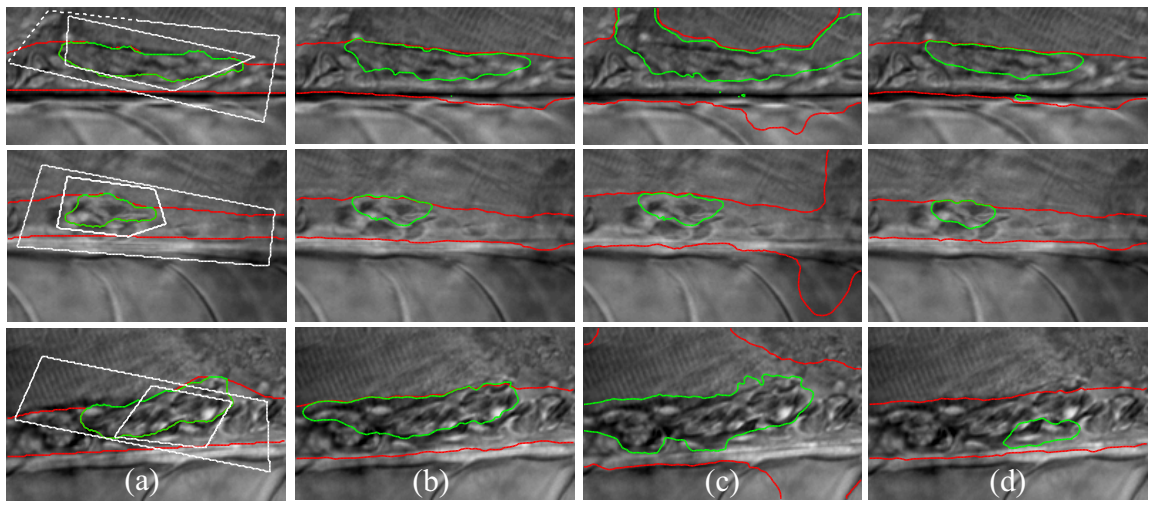

Fig. 3. (a) Ground truth and initialization; (b) Final results with our complete framework; (c)-(d) Results without the shape prior and the event-detection energies. The green contour corresponds to the thrombus, the red to the aorta.

between the boundaries of $\mathcal{C}_{12}^{i-1}$ and the estimated centerline. $E_{s p}(\omega)$ increases with $d_{R_{12}^{i-1}}^{2}(\mathbf{x})$. It thus prevents the pixels which are far away from the fitted rectangle from belonging to the thrombus $\left(\Omega_{1}\right)$ or to the aortic $\left(\Omega_{2}\right)$ regions.

\subsection{Level-Set Energy Minimization}

The three regions $\left\{\Omega_{1}, \Omega_{2}, \Omega_{3}\right\}$ are represented by two level-set functions $\Phi_{1}$ and $\Phi_{2}$. We employ the multiphase formulation [14. We define the thrombus region $\left(\Omega_{1}\right)$ by the pixels inside the two contours $\left(\Phi_{1}<0, \Phi_{2}<0\right)$, the uncoagulated aortic region $\left(\Omega_{2}\right)$ by the pixels outside the first contour and inside the second contour $\left(\Phi_{1}>0, \Phi_{2}<0\right)$, and the background region $\left(\Omega_{3}\right)$ by the pixels outside the two contours $\left(\Phi_{1}>0, \Phi_{2}>0\right)$. The complete aortic region $\left(\Omega_{12}\right)$ is directly represented by $\Phi_{2}$ and can thus be easily constrained. As suggested in [1], a topological energy $E_{\text {top }}$ is employed to constrain the number of regions in the partition (Fig 2 d). This energy penalizes the surface area of the remaining region. The weighted sum of the likelihood and prior energies forms the posterior energy

$$
E\left(\omega \mid \mathcal{S}^{p}, \mathcal{S}^{0}\right)=\alpha E_{m s}\left(\mathcal{S}^{p}, \mathcal{S}^{0} \mid \omega\right)+\beta E_{e d}\left(\mathcal{S}^{p}, \mathcal{S}^{0} \mid \omega\right)+\gamma E_{s p}(\omega)+\delta E_{t o p}(\omega),
$$

which is minimized by alternating the update of the energies with a Laplacianregularized gradient descent on $\Phi_{1}$ and $\Phi_{2}[2]$.

\section{Results}

Twenty sub-sequences taken from four microscopic video sequences and covering a wide range of biological variations (size of the thrombus, collateral vessels, etc...) were used for testing purpose. The aortic wall is artificially injured by a 


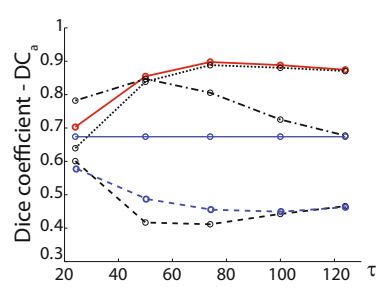

(a) Aortic vessel $\left(\Omega_{12}\right)$

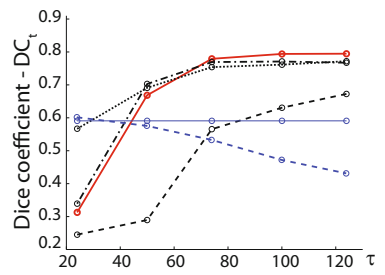

(b) Thrombus $\left(\Omega_{1}\right)$

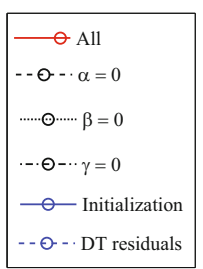

- - - - DT residuals

Fig. 4. Mean Dice-coefficient over the 20 sub-sequences, function of $\tau, \alpha, \beta, \gamma$

nitrogen-pulse ablating laser. The images are recorded with a Leica DFC420C camera through a Leica DMRXA Differential Interference Contrast (DIC) microscope equipped with a $40 \times$ water-immersed objective. The ground-truth segmentations are manually delineated by a trained biologist. The same set of empirically chosen parameters $(\alpha=1, \beta=0.25, \gamma=0.25, \delta=1)$ is used over the entire dataset. The dimensions of the DT models, $n=15$ and $m=25$, are taken from [4]. Using $\left|\Omega_{k}^{s}\right|=10$ samples per region and $\tau=75$ images of $200 \times 150$ pixels per sub-sequences, the computation time on a standard computer $(2.00 \mathrm{GHz}, 8.00 \mathrm{~GB} \mathrm{RAM})$ is of $15.5 \pm 0.25 \mathrm{~s}$ for DT model learning, of $72.4 \pm 2.05 \mathrm{~s}$ for Martin distance computation, and of $14.5 \pm 5.5 \mathrm{~s}$ for level-set evolution.

The qualitative performance of our method is illustrated in Fig. 3 . We display typical segmentation results obtained for $\tau=75$ if all the introduced energies are employed (b), if the tubular shape constraint is dismissed (c), and finally if the event-detection likelihood is discarded $(\mathrm{d})$.

The accuracy of our algorithm is quantitatively evaluated for increasing values of $\tau$ with an overlap measure, the Dice-coefficient, for both the aortic $\left(D C_{a}\right)$ and the thrombus $\left(D C_{t}\right)$ regions (cf. Fig 4). While a minimal window size is necessary to adequately capture the motion patterns, choosing the smallest possible $\tau$ prevents the temporal smoothing of the segmentation results. To this regards, $\tau=75$ is a good compromise value. Our approach yields mean DC values of $D C_{a} \geq 0.90$ and $D C_{t} \geq 0.78$. These respectively fall down to $D C_{a}=0.47(-48 \%)$ and $D C_{t}=0.57(-27 \%)$ if the DT residual energy (Fig. 1) is employed instead of the proposed energies. This shows the better performance of our method in comparison to our benchmark approach [3. We quantitatively illustrate the importance of incorporating the event-detection and the shape prior energies in addition to the motion-segmentation energy. Discarding the event-detection energy $(\beta=0)$ decreases the mean $D C_{t}$ to $0.75(-4 \%)$, and canceling the shape prior probability $(\gamma=0)$ yields a mean $D C_{a}$ of $0.80(-10 \%)$. We moreover observe in Fig 4 a that the longer the subsequence is, the more likely the perturbations in the background become. This makes the shape prior energy increasingly necessary for $\tau \geq 75$. The motion segmentation energy remains essential to obtain accurate segmentation results. If not accounted $(\alpha=0)$, the mean $D C_{a}$ decreases to $0.41(-63 \%)$ and the mean $D C_{t}$ to $0.57(-27 \%)$. 


\section{Conclusion}

In this work, we propose an innovative solution to the complex problem of joint thrombus and aortic vessel segmentation in low contrast, cluttered, and highly dynamic microscopic video sequences. By characterizing the spatial and temporal discrepancy of the motion patterns with two dynamic texture-based likelihood energies, and by constraining the aortic region with a shape constraint, we model the major facets of our biomedical imaging setting. Though applied to microscopy, we believe that the capacity of the proposed algorithm to analyze complex dynamic scenes could be beneficial to other medical imaging modalities.

\section{References}

1. Baust, M., Navab, N.: A spherical harmonics shape model for level set segmentation. In: Daniilidis, K. (ed.) ECCV 2010, Part III. LNCS, vol. 6313, pp. 580-593. Springer, Heidelberg (2010)

2. Baust, M., Zikic, D., Navab, N.: Diffusion-based regularisation strategies for variational level set segmentation. In: BMVC, pp. 19.1-19.11. BMVA Press (2010)

3. Brieu, N., Serbanovic-Canic, J., Cvejic, A., Stemple, D., Ouwehand, W., Navab, N., Groher, M.: Thrombus segmentation by texture dynamics from microscopic image sequences. In: SPIE Medical Imaging (2010)

4. Chan, A., Vasconcelos, N.: Modeling, clustering, and segmenting video with mixtures of dynamic textures. IEEE Trans. on Pattern Analysis and Machine Intelligence 30, 909-926 (2008)

5. Cremers, D., Rousson, M., Deriche, R.: A review of statistical approaches to level set segmentation: Integrating color, texture, motion and shape. Intl. J. Computer Vision 72, 195-215 (2007)

6. Dong, G., Ray, N., Acton, S.: Intravital leukocyte detection using the gradient inverse coefficient of variation. IEEE Trans. Med. Imag. 24(7), 910-924 (2005)

7. Doretto, G., Chiuso, A., Wu, Y., Soatto, S.: Dynamic textures. Intl. J. Computer Vision 51, 91-109 (2003)

8. Doretto, G., Cremers, D., Favaro, P., Soatto, S.: Dynamic texture segmentation. In: ICCV 2003, vol. 2, pp. 1236-1242 (2003)

9. Li, F., Zhou, X., Zhao, H., Wong, S.: Cell segmentation using front vector flow guided active contours. In: Yang, G., Hawkes, D., Rueckert, D., Noble, A., Taylor, C. (eds.) MICCAI 2009. LNCS, vol. 5762, pp. 609-616. Springer, Heidelberg (2009)

10. Martin, R.: A metric for ARMA processes. Trans. on Signal Processing 48(4) (2002)

11. O'Connor, M., Salles, I., Cvejic, A., Watkins, N., Ouwehand, W.: Functional genomics in zebrafish permits rapid characterization of novel platelet membrane proteins. Blood 113(19), 4754-4762 (2009)

12. Palaniappan, K., Ersoy, I., Nath, S.: Moving object segmentation using the flux tensor for biological video microscopy. In: Ip, H., Au, O., Leung, H., Sun, M., Ma, W., Hu, S. (eds.) PCM 2007. LNCS, vol. 4810, pp. 483-493. Springer, Heidelberg (2007)

13. Schmugge, S., Keller, S., Nguyen, N., Souvenir, R., Huynh, T., Clemens, M., Shin, M.: Segmentation of vessels cluttered with cells using a physics based model. In: Metaxas, D., Axel, L., Fichtinger, G., Székely, G. (eds.) MICCAI 2008, Part I. LNCS, vol. 5241, pp. 127-134. Springer, Heidelberg (2008)

14. Vese, L., Chan, T.: A multiphase level set framework for image segmentation using the mumford and shah model. Intl. J. on Computer Vision 50(3), 271-293 (2002) 